\title{
The NEXT experiment: a high-pressure xenon gas (HPGXe) TPC for the search of neutrinoless double-beta decay.
}

\section{A. Tomás* on behalf of the NEXT collaboration}

Laboratorio de Fisica Nuclear y Astroparticulas, Universidad de Zaragoza, Spain E-mail:

atomas@unizar.es

\begin{abstract}
Although neutrino oscillation experiments have shown that neutrinos have finite rest mass, their absolute mass scale is still unknown. Neutrinoless double beta decay experiments have the aim of determining the Majorana or Dirac nature of neutrinos as well as evaluating their absolute mass scale. The exploration of the degenerate hierarchy, which corresponds to an effective neutrino mass up to $50 \mathrm{meV}$, is the goal of the next generation of neutrinoless double beta decay experiments. The NEXT collaboration plans to build a $100 \mathrm{~kg}$ high-pressure Xe gas TPC enriched with ${ }^{136} \mathrm{Xe}$ for the search of the two modes of double-beta decay. Three are the features which will make this experiment very competitive in the field: an excellent energy resolution, the use of pattern recognition to reject background events and scalability to larger masses. The reasons that support this choice will be exposed as well as the detector conceptual design and the several technology options that are under consideration. The collaboration has activated a R\&D program and other activities in order to fix the most competitive approach and demonstrate its feasibility. The NEXT experiment is already approved for being installed at the main hall of the Canfranc Underground Laboratory (LSC) at 2500 m.w.e. depth in the Spanish Pyrenees.
\end{abstract}

35th International Conference of High Energy Physics - ICHEP2010,

July 22-28, 2010

Paris France

\footnotetext{
* Speaker.

${ }^{\dagger}$ For complete list of collaboration members see [2].
} 


\section{NEXT. Why to use a HPGXe TPC?}

The successful oscillation experiments have revived the search of the neutrinoless double beta decay $(0 v \beta \beta)$. While oscillations have demonstrated that the individual lepton number are not conserved and neutrinos are not zero-mass particles, the observation of $0 v \beta \beta$ would provide the absolute scale of their mass as well as determine their Majorana nature [1].

The double beta decay with neutrinos $(2 v \beta \beta)$ is a rare process but still included within the Standard Model (SM). The $0 v \beta \beta$ mode requires neutrino and antineutrino to be the same particle, so needing Physics beyond the SM. These events would appear as a peak in the spectrum situated at the $Q_{\beta \beta}$ value of the transition, that is to say, at the end of the $2 v \beta \beta$ continuous energy distribution.

NEXT stands for Neutrino Experiment with a Xenon TPC [2]. The selection of a Xe TPC is nowadays rather common in Rare Event searches [3, 4, 5]. The first reason for that is the source/target equal detector approach, which results in a monolithic detector, simpler and easy to scale up. Another important point is the fact that both ionization and scintillation can be registered. Furthermore, Xe is clean from the radiopurity point of view since it has no isotopes susceptible to be activated, and, as a noble gas, can be easily purified.

In the case of a $0 v \beta \beta$ experiment, ${ }^{136} \mathrm{Xe}$ is a $\beta \beta$-decaying isotope relatively abundant in nature, than can be concentrated by centrifugation. The $2 v \beta \beta$ mode is weak and it has never been observed by a previous experiment $\left(T_{1 / 2}^{2 v}>10^{24} \mathrm{y}\right)$. The need to discriminate the $0 v \beta \beta$ mode from the always present $2 v \beta \beta$ establishes the first obvious requirement for a $0 v \beta \beta$ experiment: a very good energy resolution. The aim of increasing the source mass in Rare Event searches drives most of the similar projects to focus in a liquid Xe TPC. To understand how NEXT intends to compensate a less dense source material by optimizing the second essential feature in a $0 v \beta \beta$ experiment, the low background, we will take a closer look of the physics case.

The $Q_{\beta \beta}$ value of $2458 \mathrm{keV}$ [6] is not only relatively high, also lies in a particular region which can result in an advantage to decrease the background. It is situated in the space between the ${ }^{208} \mathrm{Tl}$ photo-peak and its Compton edge. That means that the background level is both low as likely to be discriminated because ${ }^{208} \mathrm{Tl}$ contributions must come from complex events as energy losses by bremsstrahlung (which may be detected if interacting in the vessel' $s$ walls) or multi-Compton (presenting more than one cluster). The ${ }^{214} \mathrm{Bi} 2447 \mathrm{keV}$ gamma emission, in the neighborhood of the signal, could be the most dangerous contribution to the background. All these background events are, in the worst case, single electron tracks while the $\beta \beta$ events own a clear topological signature: two electron tracks, sharing a common vertex and finishing in a denser ionization/scintillation clouds (figure 1 ).

The above arguments suggest a powerful background rejection potential based on event topology. This idea is not new, actually it was developed by the Gotthard TPC in the 90's [7] which was able to demonstrate the rejection of 29 in 30 single electron events. In an ideal performance of this technique, $2 v \beta \beta$ mode would be the ultimate unavoidable background. Only a gas TPC can develop good enough event reconstruction capabilities. This TPC performance should be extended to a pressure as high as possible to increase the source mass. 

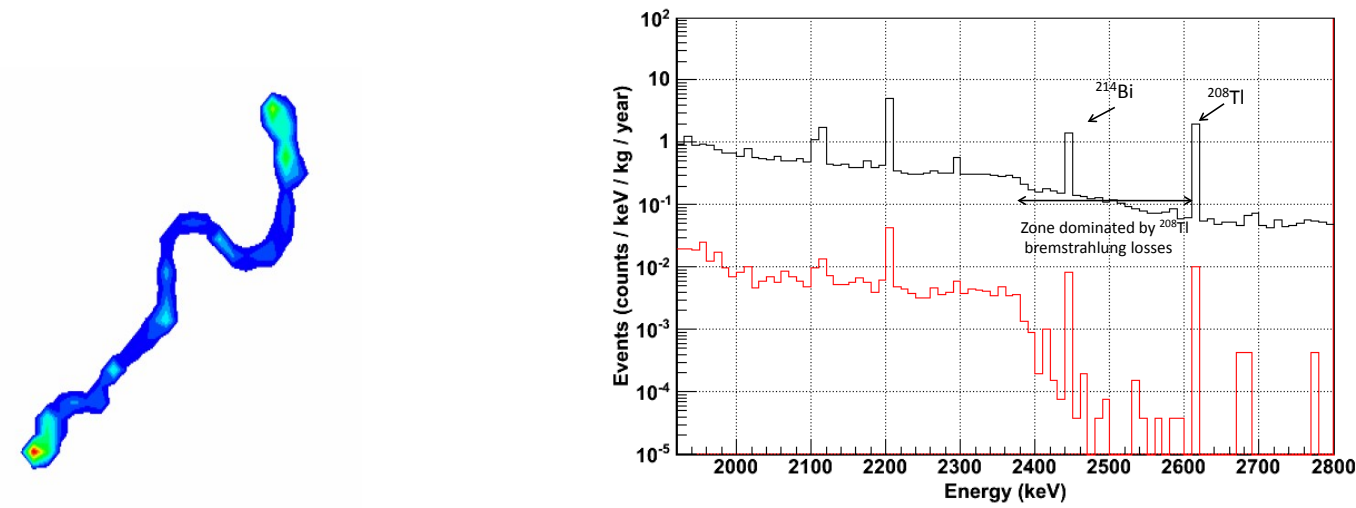

Figure 1: Left: Simulated $\beta \beta$ event showing its characteristic topological signature. Right: The comparison between simulated spectra before (in black) and after (in red) topology based cuts, illustrates the possibilities of discrimination algorithms (still under development). The RoI (around $2458 \mathrm{keV}$ ) is placed between ${ }^{208} \mathrm{Tl}$ photo-peak and its Compton-edge, very close to the ${ }^{214} \mathrm{Bi}$ peak (at $2447 \mathrm{keV}$ ). Single electron discrimination works mostly on the photo-peaks while the cuts perform better in the ${ }^{208} \mathrm{Tl}$ peak bremsstrahlung tail thanks to the discrimination of multi-cluster events.

\section{The NEXT TPC}

The NEXT project relies on the combination of high energy resolution and powerful reconstructing capabilities. The NEXT TPC must override traditional chambers' limitations and be based in the state-of-the-art readout sensors, which should extend their highlights to high pressure $\mathrm{Xe}$ gas (likely without the help of the customary quencher). All that leads to a design that is still not closed, because it is essential to choose the most practical option; while the requirements for the NEXT TPC conceptual design are very well established. It is clear, for instance, that both a primary signal (the prompt emission of VUV) and a secondary signal (produced after the drift of the ionization charges to the readout plane) must be registered. This way the difference of time between both signals allows to reconstruct the absolute position of the event so as to apply positional discrimination.

The different functions involved can be carried out by different specialized components of the TPC. PMTs will assume the tasks of registering the primary scintillation light. For the rest of the information, energy and topology, specialized, and maybe independent, readout planes can be devoted. Electroluminescense (EL) is the technique with higher potential for energy resolution in gas detectors. The light produced in the EL stage could be collected by the same PMTs used for reading the primary scintillation (the TPC must be provided of efficient reflective internal walls). Alternatively, the collaboration is exploring the performance of the amplification of charge making use of microbulk micromegas[8] (last generation of MicroPattern Gas Detectors). A matrix of photosensors behind the EL stage would carry out the tracking function. These should be SiPMs, but APDs are also being considered as well as the option of microbulk micromegas with a pixelized anode. 
The requirements projected for the NEXT detector are challenging for a TPC and need the application of latest readout technologies, sometimes themselves under development. Some time and resources are being devoted to explore directly several practical solutions in order to finally take the most suitable choice. Several medium-size (around $1 \mathrm{~kg}$ of $\mathrm{Xe}$ ) prototypes has been built so as to produce first-hand experience. There are also strong requirements of a different nature that must be considered. The already mentioned radiopurity is a particularly delicate issue. There is a program of radiopurity measurements using Ge detectors at LSC which recently gave the first measurement of a microbulk [9]. Simulations, test benches, electronics designs, studies on vessel and shielding, etc, are complementary lines of work which are already on-going [10, 11].

\section{Conclusions and prospects.}

The NEXT collaboration projects a $100 \mathrm{~kg}$ enriched Xe TPC at high pressure for a $0 \mathrm{v} \beta \beta$ experiment based in the synergy of high energy resolution and topology capabilities. During the present year several prototypes are testing different technical solutions at surface labs. At the beginning of the next year the R\&D phase should conclude with the definition of the NEXT-100 detector. One prototype will be installed at the LSC. While the data of this prototype is studied and the experience helps to prepare the site at the LSC (gas system, etc), NEXT-100 will be design and built during 2012. The experiment is planned to start running at LSC in 2013.

\section{References}

[1] H. V. Klapdor-Kleingrothaus, Sixty Years of Double Beta Decay: From Nuclear Physics to Beyond Standard Model Particle Physics", World Scientific, 2001.

[2] NEXT collaboration, Letter of Intent to the Canfranc Underground Laboratory Scientific Committee arXiv.org/0907.4054[hep-ex]

[3] C. Hall et al, Status of the EXO double beta decay search, in these proceedings.

[4] E. Aprile, T. Doke, Rev. Mod. Phys. 82 (2010) 2053.

[5] C. Hall et al, A search for weakly interacting dark matter with the LUX experiment, in these proceedings.

[6] M. Redshaw et al, Phys. Rev. Lett. 98 (2007)053003.

[7] R. Luscher et al. Phys. Lett. B434 (1998) 407-414

[8] T. Papaevangelou et al., JINST 5 (2010) P02001.

[9] S. Cebrián et al, Astropart. Phys. (2010) doi:10.1016/j.astropartphys.2010.09003

[10] L. M. P. Fernandes et al, JINST 5(2010) P09006.

[11] S. Cebrián et al, JCAP 10(2010) 010. 